\title{
Complex Treatment of the Patients with Cerebral Palsy Using Fetal Stem Cells
}

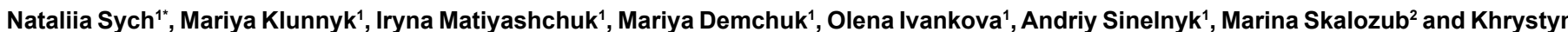
Sorochunska ${ }^{3}$

${ }^{1}$ Clinical Department, Cell Therapy Center EmCell, Kyiv, Ukraine

'Laboratory and Biotechnology Department, Cell Therapy Center EmCell, Kyiv City, Ukraine

${ }^{3}$ Stem Cells Bank, Cell Therapy Center EmCell, Kyiv, Ukraine

\begin{abstract}
Objective: The aim of this study was to examine fetal stem cells (FSCs) treatment safety and efficacy in complex therapy for cerebral palsy (CP) children. We studied clinical, neurological, and laboratory changes in CP patients before and after FSCs therapy.

Materials and methods: 11 children diagnosed with CP were under observation in Cell Therapy Center EmCell including 6 boys aged from 2 to 13 years, whose mean age was $4.23 \pm 0.24$ yrs. and 5 girls aged from 2.5 to 12 years with mean age which constituted $3.92 \pm 0.15 \mathrm{yrs}$. The patients were allocated to the Main Group (MG) who along with standard treatment received FSCs. Control group (CG) included 9 patients suffering from CP who underwent treatment solely with inclusion of conventional methods and consisted of 4 males (aged from 3 to 14 yrs. whose average age made up $4.01 \pm 0.12 \mathrm{yrs}$.) and 5 females in age from 3 to $15 \mathrm{yrs}$. with mean age by $3.87 \pm 0.18 \mathrm{yrs}$. FSCs were administered as a complex therapy for the patients with $\mathrm{CP}$ and the data of clinical and laboratory investigations were analyzed during the whole study period.
\end{abstract}

Results: It has been identified that patients who were administered treatment by use of FSCs revealed improvements of their immune status.

Conclusion: FSCs use in complex treatment of patients with CP stabilizes disease compensation and leads to improvement of immune blood findings.

Keywords: Children cerebral palsy; Fetal stem cells; Blood immunology profile; Congenital disease

Abbreviations: CP: Cerebral Palsy; WHO: world Health Organization; DNA: Deoxyribonucleic Acid; BMMC: Bone Marrow Mononuclear Cells; GMFCS: Gross Motor Function Classification System; NK: Natural Killers; HBV: Hepatitis B Virus; HCV: Hepatitis C Virus; CMV: Cytomegalovirus; DMSO: Dimethyl Sulphoxide; CFU: Colony-Forming Units; SD: Standard Deviation; CNS: Central Nervous System

\section{Introduction}

Practically one can hardly find a country all over the world without incidence of Cerebral Palsy (CP) disease among children. Simultaneously, American Academy for Cerebral Palsy research established that this disease increased prevalence rates are also stipulated by broadly implemented achievements in contemporary medicine (highly advanced technologies of maternity obstetrics in particular). Establishment of the concept from WHO-"500 g viable fetus" definitely contributed to the above statistics. Advanced technologies can be tangent to this in a certain manner too. Prominent technologies became relevant both to medical and social rehabilitation of children with CP. However, unfortunately, medical rehabilitation significantly lags behind a social one [1].

In the book of "Neural diseases" (Moscow, 1930) in the chapter devoted to cerebral palsy problems it was emphasized: "Such illness involves all cases of brain diseases which appeared as early as during intrauterine life, either at the moment of childbirth or in early childhood. Anatomy substrate of this disease: affection of the brain at time of complex maternal delivery and various organically dark diseases of embryo. Clinical pattern of such can markedly differ and; by no means this cannot be fitted to a separate illness type". And it was in 1930 ! On the contrary, we are suggesting that until this day nobody knows exactly what disease is being treated!

"Cerebral palsy is regarded as developed (mainly non-progressive) abnormality of motor functions, speech and cognitive functions which can happen in child's brain revealing developmental incompetence or abnormality against the background of immune-genetic predisposition in the children" [1]. The term "children Cerebral Paralysis" (CP) integrates a group of different syndromes by clinical manifestations, which develop resulting from brain underdevelopment; its damage on different stages of ontogenesis characterized by functional inability to keep normal posture or perform voluntary movements by the child [2]. Definition of CP usually excludes progressive hereditary diseases of nervous system, as well as some metabolic defects, affection of the spinal cord and peripheral nerves [3]. At present time it is clear that the term "cerebral palsy" cannot reflect a variety and essence of existing neurology disturbances, however, it has been widely used in world literature because until present day nobody offered another term which could comprehensively characterize similar pathological conditions in children. Their integration into a nosology group allows

*Corresponding author: Dr. Nataliia Sych, Clinical Department, Cell Therapy Center EmCell, Kyiv City, Ukraine, Tel: +380688898989; E-mail: infocenter@emcell.com

Received February 28, 2017; Accepted March 09, 2017; Published March 15, 2017

Citation: Sych N, Klunnyk M, Matiyashchuk I, Demchuk M, Ivankova O, et al. (2017) Complex Treatment of the Patients with Cerebral Palsy Using Fetal Stem Cells. J Stem Cell Res Ther 7: 378. doi: 10.4172/2157-7633.1000378

Copyright: $\odot 2017$ Sych N, et al. This is an open-access article distributed under the terms of the Creative Commons Attribution License, which permits unrestricted use, distribution, and reproduction in any medium, provided the original author and source are credited. 
planning management activities which are directed at early diagnosis and treatment of CP as far as this problem has not only medical, but also social significance [4]. Disease is associated with disturbances of locomotor apparatus (paralyses, twitchings and problems with pronunciation), disturbances of equilibrium and in some cases epilepsy and intellectual impairment. Severe forms of $\mathrm{CP}$ result in profound invalidity. Incidence of $\mathrm{CP}$ in children makes up 2-3 cases per a thousand of newborns; this disease is characteristic for approximately $1 \%$ of premature neonates $[1,2]$.

Causes of CP in children can refer to various unfavorable factors during antenatal, perinatal and postnatal periods [5-7].

The principal causes of $\mathrm{CP}$ in children are related to history of brain injury in a neonate during intrauterine life and in infancy. Among such causes are: chronic hypoxia of fetus; intrauterine infections; asphyxia during labor; pre-term or rapid labor; fetal hypothermia during the primary several hours after delivery; anemia in a mother during pregnancy; smoking, alcohol use and drugs abuse during pregnancy; infections of the nervous system during the first year of life in an infant [8].

Previous studies have shown that a $225 \mathrm{~kb}$ deletion on chromosome 9p24.3 might be responsible for the initiation of CP [9]. As ANKRD15 is the only protein coding imprinted gene within this region; $\mathrm{CP}$ might be an imprinting associated human disorders. Interestingly, since expression of imprinted genes is controlled by DNA methylation and once the imprinted DNA methylation is lost, it cannot be established in the somatic stage [10], this could explain why the FSCs, which might contain the normal patterns of imprinted DNA methylation, could be a promising therapeutic regimen for the patients with $\mathrm{CP}$. Therefore, to further understand the molecule basis of $\mathrm{CP}$, it is essential to investigate the expression levels of enzymes that involved in the maintenance of DNA methylation at imprinted loci, including DNMTs, zinc finger proteins and some histone methyltransferases [11-13].

$\mathrm{CP}$ can be easily diagnosed already during early infancy, when child could hardly reach the age of 3 years. In particularly severe cases this disease diagnosis could be established in neonatal age (before 3 months). Symptoms and signs of CP are rather individual. However, one can also emphasize a range of typical symptoms of this disease: ataxia - abnormality of muscles coordination at elementary motions performing; muscles spasticity - spasm or tension in the muscles and overstrained reflexes; stepping not on a whole foot but on the forepart of it at walking; inability to support equilibrium; uneven, shaky walk; extremely trained or relaxed muscles tone. In children with inborn $\mathrm{CP}$ of severe form doctors reveal a bad posture; their body is either too relaxed or on the contrary - muscles tension is remarkable. Spinal curving, immature mandible in children as well as microcephaly are regarded as congenital defects which coexist with this illness. Such symptoms to the extent of child's development and growth could become intense or remain unchanged [14].

Since CP is an isolated nosology unit, it is difficult to make its classification. Classification of $\mathrm{CP}$ according to M. Bax has been established (M. Bax, 1984, 1990):

1. Hemiplegic (congenital and acquired) form.

2. Spastic form.

3. Tetraplegic form.

4. Athetotic form.

5. Ataxic form.

\section{Mixed type.}

$\mathrm{CP}$ is not curable disease, therefore, one can speak only about restoration therapy or rehabilitation; however, contemporary and appropriate restoration can result in significant improvement of functions which were affected by the disease. Program of restorative treatment for a child with $\mathrm{CP}$ depends on severity, character and underlying localization of symptoms as well as existence or lack of $\mathrm{CP}$ associated disturbances of hearing, eyesight, behavior, cognitive function etc. The most serious obstacles to rehabilitation of a child with $\mathrm{CP}$ are related problems with intellect and cognitive activity which interfere with adequate interaction between the patient and instructor as well as epileptic seizures which at absence of medicines control could promote a risk of life-threatening complications for a child on the background of active stimulation therapy. Nevertheless, nowadays there are special"gentle"programs of rehabilitation for epileptic children including the methods of communication with intellectually debilitated patients suffering from $\mathrm{CP}$ - that is, each patient must have own individual program for rehabilitation with consideration of his/her opportunities, needs and problems. Duration of rehabilitative treatment for the patient with CP is not limited; in this respect such a program must be flexible and make a provision for constantly changeable factors of patient's life. The main aim of rehabilitation in case of CP is adaptation of the patient to social environment and comprehensive activity of daily living.It has been known that intrauterine infections contribute to development of hypoxia and asphyxia, result in disturbances of immunogenesis and can increase frequency of acute respiratory infections during the first year of life of the newborn. Conducted studies revealed a reduction of the main sub-populations of lymphocytes: CD3, CD4, CD8, CD19, CD56 in cases of elevation of CD95 cells count which is a marker of apoptosis in children, who had suffered from severe asphyxia $[15,16]$.

Children with CP have a decrease of adaptation resources, first of all, owing to affection of regulatory structures of CNS - hypothalamus, hippocampus and cerebral cortex $[17,18]$.

Nowadays, special methods for treatment of children with CP have been established. Methods by Vojta and Bobath concept belong to universally known approached for rehabilitation. Method by Semenova has been also recognized in Europe [19] (Russia) - which consisted in use of anti-gravitation suits as well as the method after prof. Kozyavkin [20] (Ukraine) - a system of intensive neurophysiology rehabilitation.

After all future of rehabilitation of children with CP is regarded as symbiosis of medical and social rehabilitation because CP is a polyetiologic disease.

Improvement of motor, speech and cognitive functions by virtue of modern intensive technologies use for such categories of patients is considered to be the principal task of medical rehabilitation. For this reason researchers started making experiments with novel methods of treatment and stem cells therapy belongs to such methods. Stem cell treatment opens new therapeutic opportunities for children with CP; however, it is still at the early stages of studies.

For example, Wang, Tong, Hai, et al. investigated autologous bone marrow mononuclear cell transplantation for cerebral palsy treatment and demonstrated effectiveness of this therapy in children with CP.

The other scientists administered Bone Marrow Mononuclear Cells (BMMC) into the thecal sac and proved that all patients had an uneventful post-injection course and $73 \%$ of treated patients under study presented a good response in accordance with Gross Motor Function Classification System (GMFCS) scale. An average 
improvement was 1.3 levels on GMFCS scale with cognitive advantages which were also remarkable [21].

The aim of this study was to examine FSCs treatment safety and efficacy in CP children. We studied clinical, neurological, and laboratory changes in CP patients before and after FSCs therapy.

\section{Material and Methods}

Our study allocated 11 children diagnosed with CP including 6 boys in age from 2 to 13 years, with mean age of $4.23 \pm 0.24$ yrs. and 5 girls aged from 2.5 to 12 years, mean age was $3.92 \pm 0.15$ years. All of them constituted the main group (MG) where the patients along with conventional therapy were administered FSCs. Control group (CG) consisted of 9 patients with $\mathrm{CP}$ who were treated with conventional methods only (CG) including 4 males (aged from 3 to 14 years with mean age $4.01 \pm 0.12$ years) and 5 females aged from 3 to 15 years with mean age $3.87 \pm 0.18$ years. Characteristics for both groups are described in the Table 1.

Our study was conducted in accordance with good clinical practice, Helsinki declaration and ethical standards of work with embryonic tissues of human which were approved by the Ministry of Health of Ukraine.

Clinical history taking included clinical definition of disease form along with assessment of motor functions by GMFCS scale [22]; presence and marked character of epileptic seizures as well as children's ability to self-maintenance were also evaluated in the groups under study.

All children underwent general and neurologic examination, general blood tests along with immunology testing which were conducted prior to treatment, over 6 and 12 months after FSCs transplantation. These patients underwent immunology profile analyses [B-lymphocytes: CD19+, T-lymphocytes: CD3+, T-helpers: $\mathrm{CD} 4+$, T-suppressors: CD8+, natural killer (NK): CD16+, and the factor of apoptosis: CD95+] before treatment by FSCs as well as over 6 and 12 months after it. Testing was performed at EmCell specialized laboratory (Kyiv, Ukraine) by use of cytofluorimetry (wavelength 464$480 \mathrm{~mm}$, with application of cytofluorimeter FASTER 2010, Beckton Dickinson, North Ryde, NSW, Australia). Parents of all patients before treatment initiation signed their written informed consent.

\section{Stem cell procedure: donor screening and processing of sterility}

In our study we used suspensions containing FSCs which were harvested from 6- to 9-week-old human fetuses following voluntarily elective pregnancy terminations (legally available in the Ukraine). Embryonic tissues were collected on provision of a written informed

\begin{tabular}{|c|c|c|c|c|}
\hline \multirow{2}{*}{ Number } & \multirow{2}{*}{ Gender } & CP type & MG, number of & CG, number of \\
\cline { 3 - 5 } & & patients & patients \\
\hline 1 & M & Dystonic & 1 & 1 \\
\hline 2 & M & Hypotonic & 2 & 1 \\
\hline 3 & M & Hypertonic & 3 & 2 \\
\hline 4 & F & Hypertonic & 2 & 2 \\
\hline 5 & F & Hypotonic & 1 & 2 \\
\hline 6 & F & spastic-ataxic & 1 & - \\
\hline 7 & F & Dystonic & 1 & 1 \\
\hline
\end{tabular}

Table 1: Characteristic features of the patients in MG and CG. consent taken from healthy women-donors of FSCs. Fetal material was collected in surgery premises following all rules for antiseptics being placed in special containers for transporting to the laboratory for tests. Biotechnology process of preparing FSCs suspension consisted in cells extraction from different tissues of fetus (6- to 9 -week gestation embryonic liver and brain tissues received from aborted fetuses for family planning purposes), assessment of stem cells viability was performed later as well as comprehensive programmed cryopreservation; bacteriology and virology studies were performed for all suspensions.

For the purpose of safety, both women-donors and ready-made FSCs were controlled for parasites, fungi, bacterial and viral infections like human immunodeficiency virus 1 and 2, syphilis (treponema pallidum), toxoplasmosis (toxoplasma gondii), HBV, HCV, CMV, Epstein-Barr virus, herpes simplex virus 1 and 2, rubella, ureaplasma parvum, ureaplasma urealyticum, mycoplasma genitalium, and chlamydia trachomatis.

Cryopreservation was made for the suspension containing FSCs by means of cryoprotectant $5 \%$ dimethyl sulphoxide (DMSO) using 3 stage program of freezing with velocity of $1^{\circ} \mathrm{C} / \mathrm{min}$. and crystals initiation inside of the refrigerator (Ice Cube 14S, SY-LAB Geräte $\mathrm{GmbH}$ ). All suspensions were stored in liquid nitrogen at cryobank temperature of $-196^{\circ} \mathrm{C}$.

Prior to injection all flasks with suspensions were extracted from liquid nitrogen for further water bath thawing at environment temperature $+37^{\circ} \mathrm{C}$ and preparations were adjusted until appearance of their liquid phase. All following measures were sustained at room temperature by strict rules of aseptic processing. Duration of keeping of defrosted fetal liver and brain suspensions at room temperature did not exceed $10 \mathrm{~min}$. Prior to injection a calculation of stem cells viability was made using 2 methods in parallel: Goryaev chamber and Automated Cell Counter NC-100 (Nucleo Counter Type 900-004 Chemo Metec, Denmark 2010). Number of living cells after cryopreservation made up not less than $84.7 \%$. CD34+ levels were calculated by means of flow cytofluorometry (Becton Dickinson, Franklin Lakes, NJ, USA) with fluorescent tagged antibodies (Santa Cruz Biotechnology, Dallas, TX, USA).

Stem cell procedure consisted in transplantation of the suspension containing cryopreserved FSCs following a conventional premedication by use of diphenylhydramine $10 \mathrm{mg}$ infusion and prednisone $15 \mathrm{mg}$ during the 1st day whereas a specially prepared solution was prepared for the 2nd day. For the first day all children in the main group were injected preparations made of fetal liver (for drip feed I.V. infusion) in volume of $0.5-3 \mathrm{~mL}$ which contained nucleated cells count from 1.0 to $54 \times 10^{6} / \mathrm{mL}$, and values of CD34+ progenitor cells made up from 1 up to $20 \times 10^{5} / \mathrm{mLand}$ number of mixed-lineage colony-forming units (CFU) - ranged from 0.05 to $0.82 \times 106 / \mathrm{mL}$.

\section{Statistics}

Statistical analysis of the results was performed by use of Statistica v.8.0 software (StatSoft, Inc., Tulsa, OK, USA), including calculation of average and Standard Deviation (SD). Bonferroni correction test for multiple comparison and Student's $t$ testing were applied to determine statistical value of the results $(\mathrm{p}<0.05)$ which were regarded as significant.

\section{Results and Discussion}

\section{Early post-transplantation effects}

Early post-transplantation effects were remarkable for the first week 
Citation: Sych N, Klunnyk M, Matiyashchuk I, Demchuk M, Ivankova O, et al. (2017) Complex Treatment of the Patients with Cerebral Palsy Using Fetal Stem Cells. J Stem Cell Res Ther 7: 378. doi: 10.4172/2157-7633.1000378

Page 4 of 6

after treatment. Parents noticed improvement in children's behavior, better sleep quality and appetite. In children with marked spasticity of arms and legs, parents recorded its reduction.

\section{Adverse reactions and safety}

Among children who underwent treatment with FSCs we did not observe any side effects, the same as no case of complication was reported in respect to "graft-vs-host" reaction. Simultaneously, no allergy reaction was presented by children after a course of therapy.

\section{Main effects}

Among children under study, hypertonic type of $\mathrm{CP}$ prevailed (recorded in 9 children, 45\%). In $6(30 \%)$ of children we observed a hypotonic form of this disease, whereas 4 patients (20\%) were presented with dystonic form of $\mathrm{CP}$ and 1 child revealed a mixed spastic-ataxic type of such abnormality (5\%).

According to the evaluation of CP motor functions by GMFCS

\begin{tabular}{|c|c|c|}
\hline Levels by GMFCS scale & $\begin{array}{c}\text { Absolute number of CP } \\
\text { children }\end{array}$ & $\begin{array}{c}\text { Percentage from the } \\
\text { total number of } \\
\text { patients under study }\end{array}$ \\
\hline I & 2 & $10 \%$ \\
\hline II & 4 & $20 \%$ \\
\hline III & 8 & $40 \%$ \\
\hline IV & 6 & $30 \%$ \\
\hline
\end{tabular}

Table 2: Assessment of motor functions in children with CP by GMFCS scale. system all children under study suffering from $\mathrm{CP}$ were presented with III and IV levels by GMFCS scale (Table 2).

Immunology study has been conducted for children with $\mathrm{CP}$ at the in-patient's which helped to detect the main classes of lymphocytes CD3+, CD4+, CD8+, CD4/CD8, CD16+, CD19+, CD95+. As a result of this study we determined a decreased number of practically all subpopulations of lymphocytes at increased counts of CD95+ lymphocytes which is an evidence of enhanced apoptotic activity index in children under study. After treatment performed with inclusion of FSCs in the MG patients we observed that levels of T- and B-lymphocytes were stabilized already starting from the 6th month after treatment beginning, whereas in children of the CG the same results were stabilized over 12 months after treatment, that can be considered as adaptation reaction as well as immune activation induced by complex treatment using FSCs (Tables 3 and 4). Count of CD95+ lymphocytes reduced in children of the MG over 6 months after treatment and remained within normal limits over 12 months after treatment beginning; whereas in patients of the CG levels of CD95+ lymphocytes were at the high range even over 1 year after treatment. This is suggesting that FSCs are able to inhibit apoptotic activity index along with stimulation of immune reactions in children with $\mathrm{CP}$ which simultaneously can be presented by improvement of disease clinical pattern in such children.

B-lymphocytes have been known to be relevant to humoral immunity which promotes defense from infections in children; therefore, such values are reduced in children suffering from CP. Treatment with the help of FSCs transplantation favors improvement of immunogenesis and this can be remarkable for our patients.

\begin{tabular}{|c|c|c|c|c|}
\hline \multirow{2}{*}{ Parameters } & \multirow{2}{*}{$\begin{array}{l}\text { Reference Range } \\
\text { (RR) Baseline }\end{array}$} & \multirow{2}{*}{$\begin{array}{l}\text { Before FSCs } \\
\text { treatment }\end{array}$} & \multicolumn{2}{|c|}{ After FSCs treatment } \\
\hline & & & 6 months & 12 months \\
\hline B-lymphocytes - CD19+, \% & $9.00 \pm 6.00$ & $2.50^{*}$ & 10.55 & 10.20 \\
\hline T-lymphocytes - CD3+, \% & $60.00 \pm 9.00$ & $49.70^{*}$ & 52.20 & 53.20 \\
\hline T-helpers - CD4+, \% & $39.00 \pm 5.00$ & $28.60^{*}$ & 38.12 & 35.20 \\
\hline T-suppressors - CD8+, \% & $23.00 \pm 4.00$ & $18.70^{*}$ & 21.90 & 24.16 \\
\hline NK-natural killers CD16+, \% & $12.00 \pm 6.00$ & $5.60^{*}$ & 12.46 & 11.70 \\
\hline Helper-suppressor ratio CD4+/CD8+ & $2.40 \pm 0.90$ & $1.46^{*}$ & 1.97 & 1.83 \\
\hline CD95+, \% & $17-20$ & $25^{*}$ & 20 & 18 \\
\hline
\end{tabular}

Note: differences by reference rates: * $p<0.05$

Legend: MG - Main Group; RR- Reference Range; FSCs - Fetal Stem Cells

Table 3: Dynamics of immunogram parameters among children of the MG.

\begin{tabular}{|c|c|c|c|c|}
\hline \multirow{2}{*}{ Parameters } & \multirow{2}{*}{ Reference Range (RR) Baseline } & \multirow{2}{*}{ Before FSCs treatment } & \multicolumn{2}{|c|}{ After FSCs treatment } \\
\hline & & & 6 months & 12 months \\
\hline B-lymphocytes - CD19+, \% & $9.00 \pm 6.00$ & $1.89^{*}$ & $2.85^{*}$ & 11.30 \\
\hline T-lymphocytes - CD3+, \% & $60.00 \pm 9.00$ & $48.40^{*}$ & $50.20^{*}$ & 55.10 \\
\hline T-helpers - CD4+, \% & $39.00 \pm 5.00$ & $27.68^{*}$ & 39.42 & 35.34 \\
\hline T-suppressors - CD8+, \% & $23.00 \pm 4.00$ & $17.90^{*}$ & $20.70^{*}$ & 25.10 \\
\hline NK-natural killers CD16+, \% & $12.00 \pm 6.00$ & $5.40^{*}$ & 14.36 & 11.90 \\
\hline $\begin{array}{l}\text { Helper-suppressor ratio CD4+/ } \\
\text { CD8+ }\end{array}$ & $2.40 \pm 0.90$ & $1.38^{*}$ & 2.64 & 1.97 \\
\hline CD95+, \% & $17-20$ & $26^{*}$ & $23^{*}$ & $21^{*}$ \\
\hline
\end{tabular}

Note: differences by reference rates: ${ }^{*} p<0.05$

Legend: CG - Control Group; RR- Reference Range; FSCs - Fetal Stem Cells

Table 4: Dynamics of immunogram parameters among children of the CG. 
Citation: Sych N, Klunnyk M, Matiyashchuk I, Demchuk M, Ivankova O, et al. (2017) Complex Treatment of the Patients with Cerebral Palsy Using Fetal Stem Cells. J Stem Cell Res Ther 7: 378. doi: 10.4172/2157-7633.1000378

Page 5 of 6

Cerebral palsy is a debilitating condition in the child and it imposes unusual burdens on the family and society. It is the costliest among the congenital diseases because it demands use of several medical and social resources. Any scientific trial looking for drawing useful conclusions should aim at a target population that must be as homogeneous as possible in order to come up with reliable results. Also, it is important to use all checked up methods of examination and treatment for the children with CP (stem cell therapy is among them), which have gone through clinical trials and proved their safety and effectiveness in treatment of children with CP. In addition, it is clear that we require a continuous control over children who underwent FSCs treatment for a purpose of monitoring of separate adverse reactions that belongs to our next stages of follow up with CP patients.

There are likely several effective mechanisms of FSCs action involved, including regeneration of the neural cells, direct stimulation of the neurons, the endogenous stem cells as well as trophic paracrine mediators and neovascularization.

Among the potential mechanisms of FSCs treatment effect the following advantages are related to: neuronal cell replacement, blood vessel regeneration, astrocyte and microglial cells replacement, blockade of splenic release of inflammatory cells and protection of intrinsic cells.

One of the principal concepts inherent to stem cell transplantation for children with CP is that stem cells could replace the cells of damaged nervous system. Most researchers dealing with adult stem cells show only a minimal survival of the cells transplanted with few, if any, of such stem cells displaying markers/functionality of nervous tissue [23-25].

It does not mean that replacement alone would be sufficient to account for improvements in the condition under study. While embryonic or iPS cells may have somewhat greater potential for such replacement and transformation, the number of cells undergoing this process is quite limited in vivo. Even though there may be some replacement by transplanted cells, stem cells often do not develop normal processes and may not function in neuronal circuitry [26]. Thus, cell replacement as an explanation for any improvement in the models is unlikely to be the case.

Another possibility is that the transplanted cells differentiate into astrocytes [27] or microglia. How this would assist in functional recovery is unclear.

\section{Conclusions}

Our pilot study is a preliminary demonstration of safety and efficacy of FSCs administration in children with CP. Our research has shown that FSCs therapy, irrespective of CP severity, favorably influences the course of children's development and their immunological markers. Procedure of fetal stem cells transplantation is simple and non-invasive which cannot cause complications or development of allergy reactions. For making FSCs effect more intense we recommended our patients a complex treatment with inclusion of medicines and use of different additional methods. How FSCs work in CP is understood incompletely and needs to be more fully investigated. Such future profound research studies are required as well as larger randomized, placebo-controlled trials would help us to further characterize potential of FSCs associated improvements in children with CP.

\section{Acknowledgments}

All authors made a great contribution to statistic data processing and conduction of all studies on the patients with cerebral palsy and complex treatment

\section{by use of fetal stem cells.}

\section{Competing Interests}

The paper is intended to be an original paper; all authors of the manuscript are members of Cell Therapy Center EmCell, Kyiv, Ukraine. The authors have approved the manuscript and agree to its submission. There are no matters relevant to the conflict of interests among the authors who contributed to manuscript submission.

\section{References}

1. Yevtushenko SK (2014) Etiology and pathogenesis of cerebral palsy in children. International neurology journal 65: 117-123.

2. Semenova KA, Makhmudova NM (1980) Medical rehabilitation and social adaptation of children with cerebral palsy. Tashkent: Medicine p: 275

3. Badalyan LO, Zhurba LT, Timonina OV (1987) Problems of children cerebra palsy classification. Journal of neuropathology and psychiatry 10: 1445-1448.

4. Badalyan LO, Zhurba LT (1988) Cerebral palsy in children. Kyiv.

5. Yevtushenko SK, Shestova OP, Morozova TM (2003) Hypoxic brain injuries in newborns. K: Intermed p: 101

6. Dolgikh TI, Belkova TN, Tirskaya YI, Nesterenko EV, Vlasenko NY, Shevelyov MV (2011) Clinical-immunology aspects of intrauterine infections with centra nervous system damage in newborns. Cytokines and inflammation 10: 46-50.

7. Mallard C, Wang X (2012) Infection-Induced Vulnerability of Perinatal Brain Injury. Neurol Res Int 2012: 6

8. Volpe JJ (2008) Postnatal Sepsis, Necrotizing Entercolitis, and the Critical Role of Systemic Inflammation in White Matter Injury in Premature Infants. Journ Pediatr 153: 160-163.[PubMed]

9. Bugrym NV, Logvinova II (2010) Association of immune status and morbidity until 1 year in children who have suffered from severe asphyxia. Herald of novel medical technologies 18: 13-15.

10. Lerer I, Sagi M, Meiner V, Cohen T, Zlotogora J (2005) Deletion of the ANKRD15 gene at 9p24.3 causes parent-of-origin-dependent inheritance of familial cerebral palsy. Hum Mol Gen 14: 3911-3920.[PubMed]

11. Reik W, Walter J (2001) Evolution of imprinting mechanisms: the battle of the sexes begins in the zygote. Nat Rev Genet 2: 21-32.[PubMed]

12. En Li, Beard C, Jaenisch R (1993) Role for DNA methylation in genomic imprinting. Nature 366: 362-365

13. Li X, Ito M, Zhou F, Youngson N, Zuo X, et al. (2008) A maternal-zygotic effect gene, Zfp57, maintains both maternal and paternal imprints. Dev Cell15: 547557.[PubMed]

14. Zhang T, Termanis A, Özkan B, Bao XX, Culley J, et al. (2016) G9a/GLP Complex Maintains Imprinted DNA Methylation in Embryonic Stem Cells. Cell Reports 15: 77-85.[PubMed]

15. Yevtushenko SK, Yevtushenko OS, Dubovtseva OO (2001) The principal ten causes affecting growth of organic nervous system diseases in children, their management possibility by medical staff. Social paediatrics 2001: 287-289.

16. Kryvushchev BI, Yulish El (2009) Methods of rehabilitation of sickly children who had suffered from perinatal damage of central nervous system. Health of a Child 1: 25-28

17. Yulish YI. Significance of autoimmune processes in development of children with perinatal damage of central nervous system. Health of a Child 2: 42-47.

18. Gibson CS, Maclennan AH, Dekker GA, Goldwater PN, Sullivan TR, et al. (2008) Candidate Genes and Cerebral Palsy: A Population-Based Study. Pediatrics 122: 1079-1085.[PubMed]

19. O'Callaghan ME, MacLennan AH, Haan EA, Dekker G (2009) The genomic basis of cerebral palsy: a HuGE systematic. Literature review. Human Genetic 126: $149-172$.

20. Semenova KA (2007) Restorative treatment for children with perinatal affection of nervous system and cerebral paralysis in children. Law and order P: 612.

21. Kozyavkin VI, Sak NN, Kachmar OA, Babadagly MA (2007) Principles of rehabilitation of motor disturbances by Kozyavkin method. Lviv: NEH Ukrainian technologies P: 192.

22. Nassim HAC, Tarek WW, Ramzi A, et al. (2016) Bou Habib Treatment of 
Citation: Sych N, Klunnyk M, Matiyashchuk I, Demchuk M, Ivankova O, et al. (2017) Complex Treatment of the Patients with Cerebral Palsy Using Fetal Stem Cells. J Stem Cell Res Ther 7: 378. doi: 10.4172/2157-7633.1000378

Cerebral Palsy with Stem Cells: A Report of 17 Cases. Int J Stem Cells 9: 9095.[PubMed]

23. Palisano R, Rosenbaum P, Walter S, Russell D, Wood E, et al. (1997) Development and Reliability of a System to Classify Gross Motor Function in Children with Cerebral Palsy. Dev Med Child Neurol 39: 214-223.[PubMed]

24. Yasuhara T, Hara K, Maki M, Mays RW, Deans RJ, et al. (2008) Intravenous grafts recapitulate neurorestoration afforded by intracerebrally delivered multipotent adult progenitor cells in neonatal hypoxic-ischemic rats. J Cereb Blood Flow Metab 28: 1804-1810.[PubMed]
25. Reiss P, Zhang C, Saatman K, Laurer HL, Longhi LG, et al. (2002) Transplanted neural stem cells survive, differentiate, and improve neurological motor function after experimental traumatic brain injury. Neurosurgery 51: 1043-1052. [PubMed]

26. Zhao LR, Duan WM, Reyes M, Keene CD, Verfaillie CM, et al. (2002) Human bone marrow stem cells exhibit neural phenotypes and ameliorate neurological deficits after grafting into the ischemic brain of rats. Exp Neurol 174: 11-20. [PubMed]

27. Kopen GC, Prockop DG, Phinney DG (1999) Marrow stromal cells migrate throughout forebrain and cerebellum, and they differentiate into astrocytes after injection into neonatal mouse brains. Proc Natl Acad Sci U S A 96: 1071110716.[PubMed] 